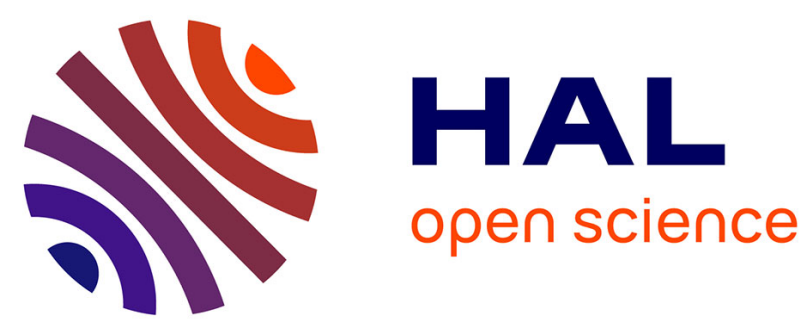

\title{
POINT-SPREAD FUNCTION MODEL FOR FLUORESCENCE MACROSCOPY IMAGING
}

Praveen Pankajakshan, Zvi Kam, Alain Dieterlen, Gilbert Engler, Laure Blanc-Féraud, Josiane Zerubia, Jean-Christophe Olivo-Marin

\section{To cite this version:}

Praveen Pankajakshan, Zvi Kam, Alain Dieterlen, Gilbert Engler, Laure Blanc-Féraud, et al.. POINTSPREAD FUNCTION MODEL FOR FLUORESCENCE MACROSCOPY IMAGING. Forty-fourth asilomar conference on signals, systems and computers, IEEE Signal Processing Society, Nov 2010, Monterey, United States. inria-00555940

\section{HAL Id: inria-00555940 \\ https://hal.inria.fr/inria-00555940}

Submitted on 14 Jan 2011

HAL is a multi-disciplinary open access archive for the deposit and dissemination of scientific research documents, whether they are published or not. The documents may come from teaching and research institutions in France or abroad, or from public or private research centers.
L'archive ouverte pluridisciplinaire $\mathbf{H A L}$, est destinée au dépôt et à la diffusion de documents scientifiques de niveau recherche, publiés ou non, émanant des établissements d'enseignement et de recherche français ou étrangers, des laboratoires publics ou privés. 


\title{
POINT-SPREAD FUNCTION MODEL FOR FLUORESCENCE MACROSCOPY IMAGING
}

\author{
Praveen Pankajakshan ${ }^{* 1}$, Zvi Kam ${ }^{2}$, Alain Dieterlen ${ }^{3}$, Gilbert Engler ${ }^{4}$, \\ Laure Blanc-Féraud ${ }^{5}$, Josiane Zerubia ${ }^{5}$, Jean-Christophe Olivo-Marin ${ }^{* 1}$ \\ ${ }^{1}$ Quantitative Image ${ }^{2}$ Molecular Cell Biology, \\ Analysis Unit, \\ Institut Pasteur, \\ 75015 Paris, France. \\ ${ }^{3}$ Laboratoire \\ MIPS-LAB.EL, \\ Science, \\ Rehovot 76100, Israel. \\ ${ }^{4}$ IBSV Unit, \\ INRA, \\ Université de Haute-Alsace, 06903 Sophia Antipolis, \\ 68093 Mulhouse, France. \\ ${ }^{5}$ ARIANA Project-team, \\ INRIA/CNRS/UNS, \\ 06902 Sophia Antipolis, \\ France.
}

\begin{abstract}
In this paper, we model the point-spread function (PSF) of a fluorescence MACROscope with a field aberration. The MACROscope is an imaging arrangement that is designed to directly study small and large specimen preparations without physically sectioning them. However, due to the different optical components of the MACROscope, it cannot achieve the condition of lateral spatial invariance for all magnifications. For example, under low zoom settings, this field aberration becomes prominent, the PSF varies in the lateral field, and is proportional to the distance from the center of the field. On the other hand, for larger zooms, these aberrations become gradually absent. A computational approach to correct this aberration often relies on an accurate knowledge of the PSF. The PSF can be defined either theoretically using a scalar diffraction model or empirically by acquiring a three-dimensional image of a fluorescent bead that approximates a point source. The experimental PSF is difficult to obtain and can change with slight deviations from the physical conditions. In this paper, we model the PSF using the scalar diffraction approach, and the pupil function is modeled by chopping it. By comparing our modeled PSF with an experimentally obtained PSF, we validate our hypothesis that the spatial variance is caused by two limiting optical apertures brought together on different conjugate planes.
\end{abstract}

Index Terms - fluorescence MACROscopy, point-spread function, pupil function, vignetting.

\section{INTRODUCTION}

In recent years, imaging techniques have emerged in all areas of life sciences in order to study basic cellular, biochemical, physiological and pathological processes. Innovative imaging technologies are deployed to produce images of biological systems at the cellular, organ and whole body levels, either independently or in combination. Although, the last decade has seen a growth in new imaging systems involving smaller samples (e.g. fluorescent microscopes), fluorescent MACROscopes [see 1] were recently commercialized that combines the best of MACROscope and a microscope. This imaging system collects emitted fluorescence from relatively large samples (up to a couple of centimeters), in three-dimensions (3-D), and also can perform time-lapse imaging. A fluorescence MACROscope works on

This research was supported by the ANR DIAMOND project (http://www-syscom.univ-mlv.fr/ANRDIAMOND/). The authors gratefully acknowledge Dr. Philippe Herbomel (Institut Pasteur, France) and Dr. Didier Hentsch (Imaging Centre, IGBMC, France) for letting us use their MACROscopes and for the interesting discussions. We would also like to thank Dr. Mickael Lelek (Institut Pasteur, France) for preparing the bead samples.

*Email: \{praveen,jcolivo\}@ pasteur.fr. the same principle as of a microscope, except for an additional zoom lens or lenses in the MACROsope ( $c f$. Fig. 1). This extra accessory to the optical system allows observation of large object fields and to image at relatively larger working distances, which means going deeper into tissues. By fixing the objective and changing the zoom

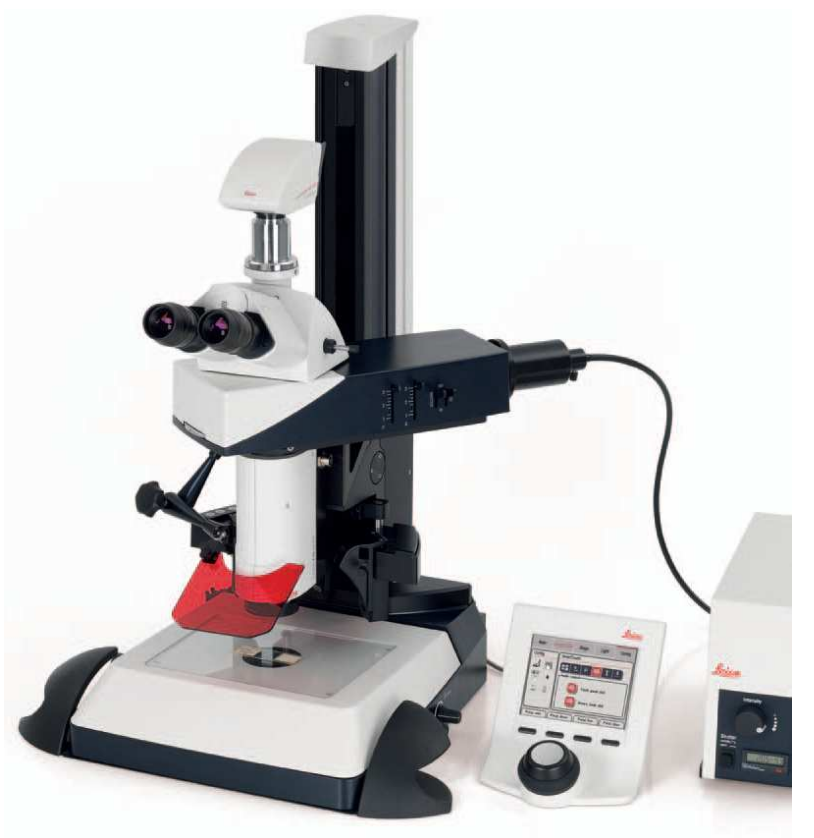

Fig. 1. A commercial MACROscope, the Leica MacroFluo ${ }^{\mathrm{TM}}$ Z16APO.

positions, the system can work at a distance of about $80 \mathrm{~mm}$ above the specimen and provide a $20 \mathrm{~mm}$ (diagonal diameter) field-of-view (FOV).

It is primarily important to measure and accurately model the point-spread function (PSF) of any optical system. This exercise can detect the different type of aberrations which can affect the images, and eventually help in correction by either modifying the arrangement or computationally. To our best knowledge, this is the first time that the MACROscope PSF is characterized or modeled by taking into account the associated field aberration. The only prior work in this field is on computationally correcting the field aberrations that occur in consumer digital cameras [see 2; 3; 4]. These methods either use a parametric model for the aberration and estimate the parameters, or use a general smoothing term for the aberration func- 
tion while restoring the image simultaneously. While these models and restoration techniques might be effective for two-dimensional (2-D) images, when it comes to 3-D fluorescence data, it is not applicable. This is because the problem of field aberration is coupled with the problem of out-of-focus blur, in the z-direction, due to the diffraction-limited nature of the lens [see 5].

This article is organized as follows. In section 2, we discuss the field aberrations in general and more specifically optical vignetting in photography and in a MACROscope. This is followed by a short section on the determination of the PSF empirically. An analysis on the extension of a scalar PSF model to fluorescence MACROscopy is discussed in section 2.3, followed by the numerical simulation and experimental validation in section 3 .

\section{FIELD ABERRATIONS}

In most lens systems, the finite length of the tube/barrel between the entrance and the exit apertures causes the oblique rays to be cut off gradually as the obliquity is increased. The obliquely incident light is confronted with a smaller lens opening than the light that is approaching the lens head-on (cf. Fig. 2). Eventually, for the ex-

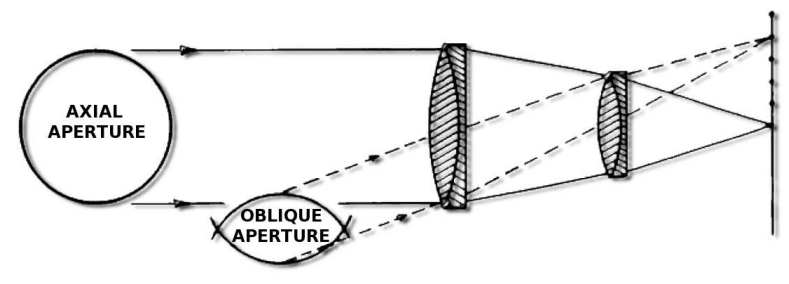

Fig. 2. The vignetting of an oblique light beam in a lens. Reproduced from [6]

treme oblique rays, the light is extinguished altogether. Vignetting, as this phenomenon is known in photography, is the denotation of the gradual fading of the intensity of an image from the centre towards the edge. As a result, an unintended darkening of the image corners might appear in a photographic image. While natural and optical vignetting might be inherent to the optical lens system design, mechanical vignetting is usually caused by due to protrusions that partially occlude the FOV [7]. The difference between them is that while natural and optical vignetting leads to a gradual transition from a brighter image center to darker corners, for mechanical vignetting, the fall-off is usually abrupt. In this paper, we will only discuss optical vignetting as this is the primary field aberration in a MACROscope. An important remark is that the optical vignetting should not be confused with the Petzval field curvature, an optical aberration, caused due to the inability of the lens to focus a flat object normal to the optical axis to a flat image plane.

In Fig. 3, we illustrate optical vignetting for the Pacific Grove beach, CA, USA, taken using a Nikon COOLPIX P7000 camera, at $f / 2.8$ and $f / 3.5$. At the full aperture setting, the image reveals a 'hot spot': a brighter center and a darkening towards the corners (left photograph). When the aperture is closed down to $f / 3.5$, the light fall-off has disappeared, and a relatively evenly illuminated beach is revealed (right photograph). At the setting $f / 2.8$, the aperture for off-axis is markedly reduced compared to the on-axis case as the entrance pupil is partially shielded by the lens barrel. More precisely, the aperture is delimited by the rim surrounding the front element. For a smaller aperture, the lens collects less light for the
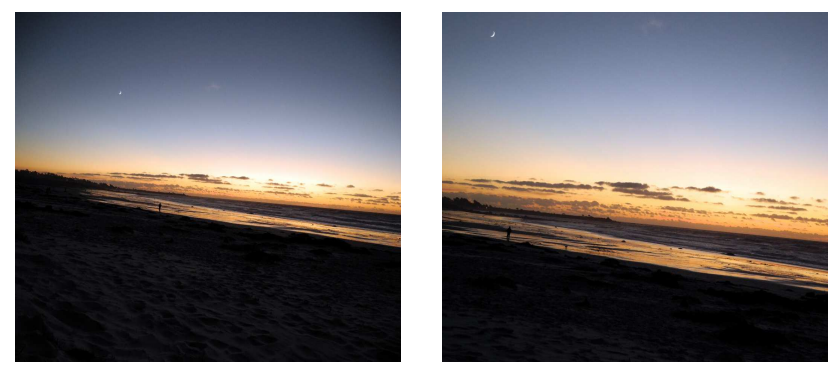

Fig. 3. Left: Optical vignetting at $f / 2$.8. Right: no vingetting at f/3.5. (Photograph by Praveen Pankajakshan.)

off-axis points than for the on-axis points, and hence the image corners will be darker than the image center. At the setting $f / 3.5$, the entrance pupil is much smaller and no longer shielded by the lens barrel. Consequently, obliquely incident light sees the same aperture as normally incident light and there is no optical vignetting.

\subsection{Cat's eye effect and aperture chopping}

The consequences of optical vignetting for a subject that is in focus is merely a reduced brightness towards the image corners. However, optical vignetting can also have a pronounced effect on the out-of-focus parts of the image. This is because, the shape of the out-of-focus highlight $(\mathrm{OOFH})$ mimics the shape of the clear aperture. For example, in Fig. 4, the bottom left section of the image is the part which is in-focus, while the middle and the upper right corners are the OOFH. From the enlarged rectangular inset, we see that the OOFH shows distant point sources having a cat's eye effect. In photography, the quality of the picture can be judged by the way the lens renders the out-of-focus points of light. This blur or bokeh can have the shape of the aperture and can sometimes be intentionally added for aesthetic reasons. In a MACROscope however, this is not desirable. With an increasing distance from the optical axis, the shape of the OOFH progressively narrows and starts to resemble a cat's eye, hence the name. The larger the distance from the image center, the narrower the cat's eye becomes. By judging the narrowness of the cat's eye with an OOFH in the image corner, it is possible to estimate the amount of optical vignetting. This effect tends to be stronger in wideangle and large aperture lenses, but the effect can be noticed in most photographic lenses. Zoom lenses are often saddled with a fair amount of optical vignetting. Oversized front or rear elements help to reduce this type of vignetting and are frequently applied in wideangle lens designs.

\subsection{Empirically derived Point-Spread Function}

Fig. 1 shows a MacroFluo ${ }^{\mathrm{TM}} \mathrm{Z16APO}$ from Leica. This MACROscope is equipped with two objective lenses of magnification $2 \times$ and $5 \times$. The zoom factor is 16 , with positions selectable between $0.57 \times-9.2 \times$. Unlike a stereomicroscope, it is claimed that the vertical beam path of the MACROscope allows parallax free imaging while making z-series stacks. This MACROscope is also equipped with a motorized column for precise axial focus that can be controlled with the software MacroVue $\mathrm{e}^{\mathrm{TM}}$ or by using the SmartTouch $^{\mathrm{TM}}$. It is fit with a highly sensitive cooled digital color camera, CoolSNAP ${ }^{\mathrm{TM}} \mathrm{HQ}$, and the operating mode could be changed to take monochrome or multi-channel images as the case maybe. Each of the experiment performed could be recorded in a 


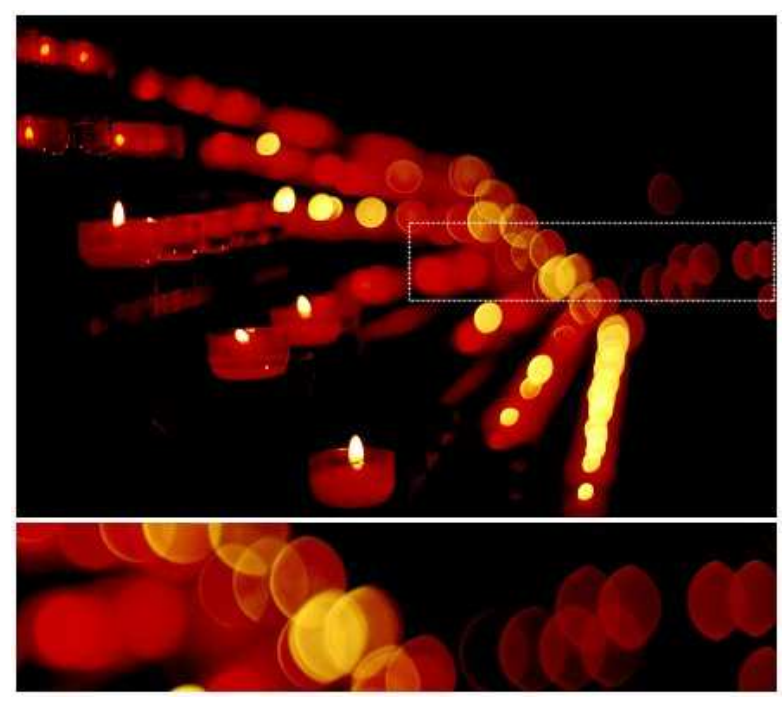

Fig. 4. The cat's eye effect. The rectangular area indicated by the dotted white line is shown enlarged at the bottom. (Photograph by Peter Boehmer.)

separate journal file that permits the user to acquire images under the same settings automatically without any need for fine tuning. The coverslip is of type 1.5 , and has a thickness of $170 \mu \mathrm{m}$ and refractive index (RI) of 1.522.

The optical resolution of a MACROscope (widefield) in the lateral and the axial direction should be calculated to know the size of the point source to be imaged. This resolution is given by the Rayleigh criterion [see 8] as:

$$
\begin{gathered}
r_{\text {lateral }}=0.61 \frac{\lambda_{\mathrm{em}}}{\mathrm{NA}}, \\
r_{\text {axial }}=0.885 \frac{\lambda_{\mathrm{em}}}{n_{i}-\left(n_{i}^{2}-\mathrm{NA}^{2}\right)^{\frac{1}{2}}},
\end{gathered}
$$

where $\lambda_{\mathrm{em}}$ is the wavelength of the emitted light, $n_{i}$ is the RI of the objective immersion medium (here 1.00 for air), and NA is the effective numerical aperture. If the distance between two closely spaced point sources is lesser than $r_{\text {lateral }}$ in the lateral or $r_{\text {axial }}$ in the axial direction, then they cannot be resolved.

For small NA, $\left(n_{i}^{2}-\mathrm{NA}^{2}\right)^{1 / 2}$ can be approximated in the following way by using Binomial expansions:

$$
\begin{aligned}
r_{\mathrm{axial}} & \approx 0.885 \frac{\lambda_{\mathrm{em}}}{n_{i}-n_{i}\left(1-\frac{\mathrm{NA}^{2}}{2 \times n_{i}^{2}}\right)}, \\
& =\frac{0.885 \times 2 \times n_{i} \times \lambda_{\mathrm{em}}}{\mathrm{NA}^{2}}, \\
& =1.77 \frac{n_{i} \times \lambda_{\mathrm{em}}}{\mathrm{NA}^{2}} .
\end{aligned}
$$

Since the emission spectrum is in the green side, the emission peak wavelength is $\lambda_{\mathrm{em}}=520 \mathrm{~nm}$, when the excitation peak is at $\lambda_{\mathrm{ex}}=488 \mathrm{~nm}$. As the maximum NA is 0.5 , the minimum possible resolvable structure is of size $0.63 \mu \mathrm{m}$ along the lateral plane and $3.68 \mu \mathrm{m}$ along the axial direction.
Direct measurement of a widefield MACROscope PSF requires the object size to be sub-resolution. Here, subresolution refers to an object whose dimensions are smaller than the diffraction limit of the MACROscope objective. Fluorescent objects of this size yield a weak signal that requires an extremely sensitive detector and limits the axial range over which the PSF can be measured. We used polystyrene latex microspheres from Tetraspeck ${ }^{\mathrm{TM}}$ with the manufacturer specified diameter of about $250 \mathrm{~nm}$. These spheres have a peak excitation/emission wavelengths of 505/515nm (green). Due to the enormous difference in the resolution between the lateral and the axial directions (a factor of about 6 ), the imaged microspheres are relatively small along the axial direction.

There are several methods available in literature for experimentally imaging sub-resolution microspheres; the notable ones are in $[9 ; 10]$. In these, a series of 2-D images of a sub-resolution object with different amounts of defocus are taken. For our experiment, we used a very simple imaging setup. Some fluorescent microspheres are dried on to the bottom of a coverslip, and the coverslip is placed in the immersion medium. Fig. 5 shows the schematic of the experiment. The axial sampling was fixed at $1 \mu \mathrm{m}$ and the radial sampling

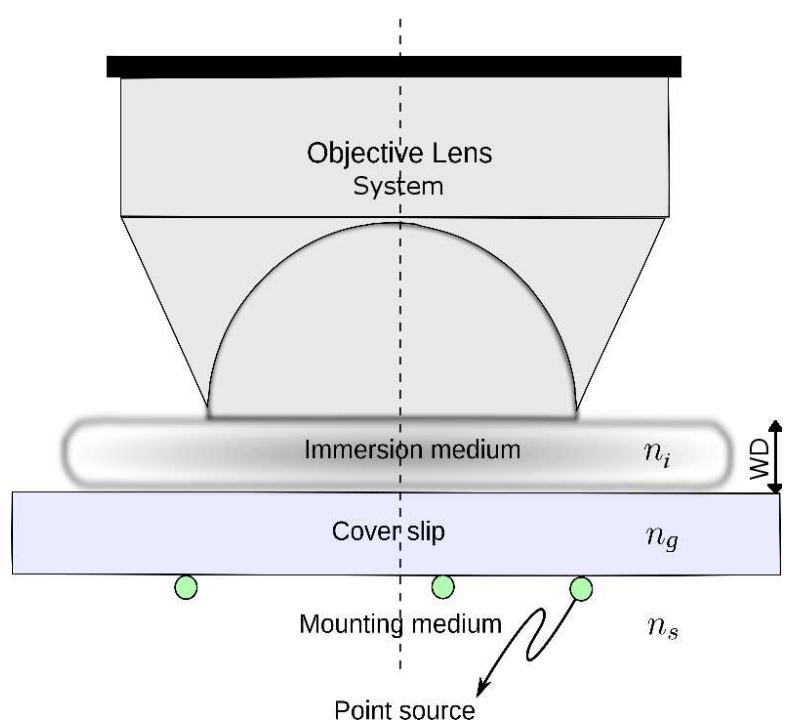

Fig. 5. A schematic of the experimental set-up for imaging subresolution microbeads for determining the PSF. For a MACROscope, the medium between the lens and the cover slip is usually air, so the RI, $n_{i}=1.0$, and the mounting medium RI, $n_{s} \approx n_{g}=1.518$.

varies with the zoom positions. Fig. 6 shows the maximum intensity projection of the imaged beads along the lateral axis. The zoom is set at about $1.6 \times$. For this zoom position, the radial sampling was $998.3 \mathrm{~nm}$. We notice that the image obtained is different in the different positions of the lateral field.

\subsection{Scalar PSF model}

In order to understand the vignetting in a MACROscope, we look at the scalar diffraction model of the magnitude PSF, $h(x, y, z)$, where $(x, y, z) \in \Omega_{S}$ are the spatial coordinates. From the KirchhoffFraunhofer approximation [see 8], we can write the near-focus amplitude PSF, $h_{\mathrm{A}}(x, y, z)$, in terms of the inverse Fourier transform of 


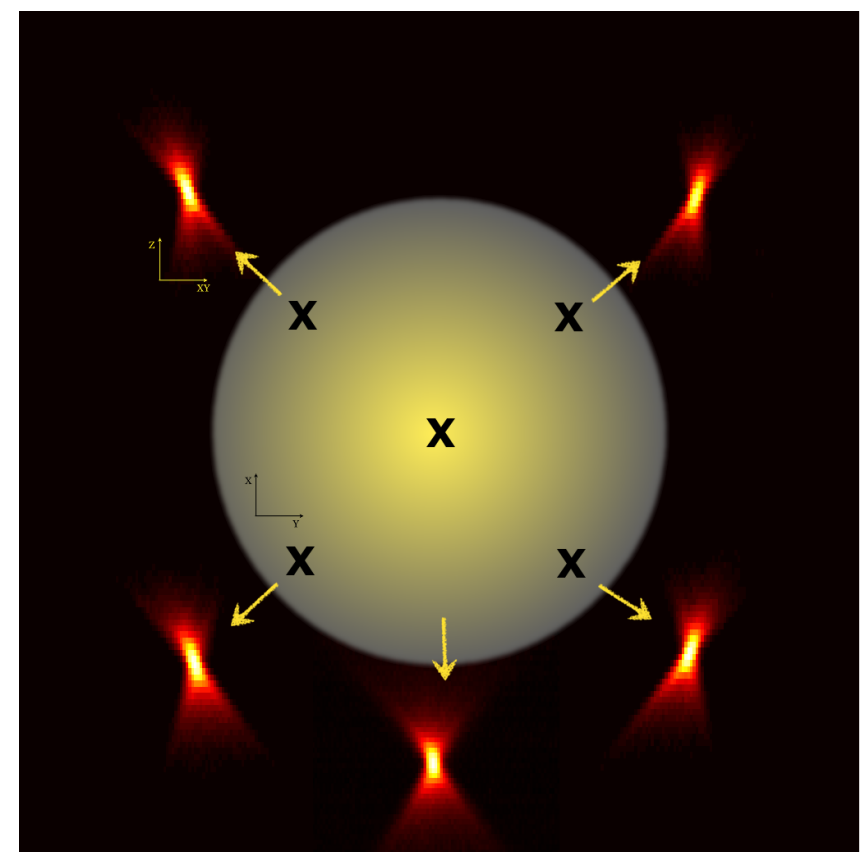

Fig. 6. Variation of bead images with lateral position in the object field. The five different lateral positions are shown on the lateral field while the bead images are the maximum intensity projections along their lateral axes.

the 2-D exit pupil function, $P\left(k_{x}, k_{y}, z\right)$, at each defocus $z$ as:

$$
h_{\mathrm{A}}(x, y, z)=\mathcal{F}_{2 \mathrm{D}}^{-1}\left\{P\left(k_{x}, k_{y}, z\right)\right\},
$$

where $\left(k_{x}, k_{y}, k_{z}\right) \in \Omega_{f}$ are the coordinates in the pupil domain. The above expression states that the field distribution of a point source in an image space is the inverse Fourier transform of the overall complex field distribution of the wavefront, in the back pupil plane of the optical system. We also notice that the inverse Fourier transform of an unaberrated pupil, when considered as a circular disc, gives the Airy disc pattern (Bessel function). If we represent $\left(k_{x}, k_{y}\right)$ in the radial coordinates, $\theta_{i}=\arcsin \left(\left(k_{x}^{2}+k_{y}^{2}\right)^{1 / 2} / k_{i}\right)$ and $k_{i}=2 \pi n_{i} / \lambda_{\text {ex }}$. The pupil function of the objective, $P_{\text {obj }}\left(k_{x}, k_{y}, z\right)$, after including defocus, can be written as:

$$
P_{\mathrm{obj}}\left(k_{x}, k_{y}, z\right)= \begin{cases}\exp \left(j k_{0} \varphi\left(\theta_{i}, \theta_{s}, z\right)\right), & \text { if } \frac{\left(k_{x}^{2}+k_{y}^{2}\right)^{\frac{1}{2}}}{k_{i}}<\frac{\mathrm{NA}_{\mathrm{obj}}}{n_{i}} \\ 0, & \text { otherwise }\end{cases}
$$

where $\mathrm{NA}_{\mathrm{obj}}$ is the numerical aperture of the objective lens, and $\varphi\left(\theta_{i}, \theta_{S}, z\right)$ is the optical phase difference between the wavefront emerging from the exit pupil and a reference wave. In the above expression, the amplitude of the pupil function is approximated to be a constant. $\theta_{S}$ is the angle in the object plane related to $\theta_{i}$ by Snell's law as $n_{i} \sin \theta_{i}=n_{s} \sin \theta_{s}$. The overall pupil function of the entire optical arrangement is given in Eq. (6), where $\mathrm{NA}_{\mathrm{z}}$ is the numerical aperture of the zoom lens system. In this expression, the parameters $\left(r_{x}, r_{y}\right)$ gives the relative displacement between the two apertures as is shown in Fig. 7. The aperture overlap region permits the light while the rest blocks it out. It can be seen that when $\left(r_{x}, r_{y}\right)$ decreases, the displacement between them is reduced

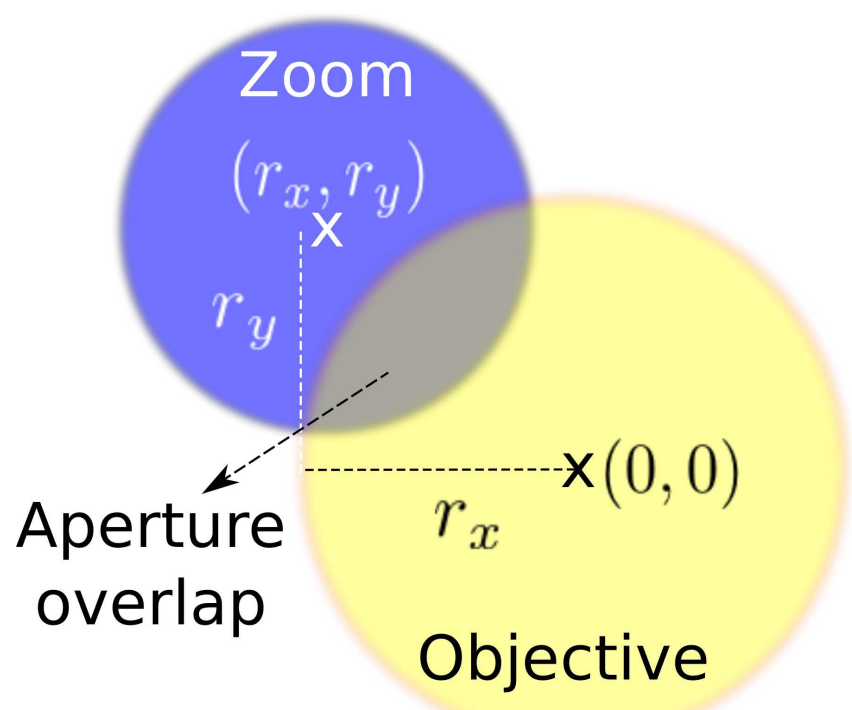

Fig. 7. Overlap between two apertures for the pupil function calculation. This overlap region between the two apertures is the light permitting region.

and more light is collected/permitted. In the limiting case, when $\left(r_{x}, r_{y}\right)=(0,0)$, the entire light passes through. For light to pass through, $\left(r_{x}^{2}+r_{y}^{2}\right)^{1 / 2}<\left(r_{\mathrm{z}}+r_{\mathrm{obj}}\right)$, where $r_{\mathrm{z}}$ and $r_{\mathrm{obj}}$ are the radii of the zoom and the objective apertures respectively. On the other hand, when $\left(r_{x}^{2}+r_{y}^{2}\right)^{1 / 2} \geq\left(r_{\mathrm{z}}+r_{\mathrm{obj}}\right)$, no light passes through.

The phase $\varphi\left(\theta_{i}, \theta_{s}, z\right)$, in Eq. (5), is the sum of the defocus term $\varphi_{d}\left(\theta_{i}, z\right)$ and the aberration phase $\varphi_{a}\left(\theta_{i}, \theta_{S}\right)$. If we ignore all other aberrations except vignetting, then the phase term expression is given by only the defect of focus as [see 11]:

$$
\varphi_{d}\left(\theta_{i}, z ; n_{i}\right) \approx z\left(1-\cos \theta_{i}\right) \text {. }
$$

In the widefield case, the emission amplitude PSF, $h_{\mathrm{A}}\left(\mathbf{x} ; \lambda_{\mathrm{em}}\right)$, could be assumed to be close to the excitation amplitude PSF, $h_{\mathrm{A}}\left(\mathbf{x} ; \lambda_{\mathrm{ex}}\right)$, so that they are related to the magnitude PSF, $h(\mathbf{x})$, by

$$
h(\mathbf{x})=\left|h_{\mathrm{A}}\left(\mathbf{x} ; \lambda_{\mathrm{ex}}\right)\right|^{2} .
$$

As the MACROscope works under a low NA, the effect of polarization can be neglected, and a scalar diffraction model is appropriate.

\section{RESULTS AND DISCUSSION}

In Fig. 8, the chopped pupil is simulated by using the Eqs. (5) and (6), for a Leica MacroFluo ${ }^{\text {TM }}$ Z16APO. The colored region shows the amount of light permitted and this corresponds to only $25 \%$ of the maximum light flux. By modifying the amount of overlap, we can model the PSF in the different positions along the lateral field. If we look at the corresponding magnitude PSF in Fig. 9 that is obtained from the above $75 \%$ chopped pupil, by using Eqs. (4) and (8), we notice that the far side out-of-focus slice has the same shape as the aperture in Fig. 8. We compare this theoretically modeled PSF in Fig. 10, for an aperture overlap of about $25 \%$, with an empirically obtained image of a $4 \mu \mathrm{m}$ fluorescent microsphere. The zoom position for this experimentally obtained image is $1.6 \times$ and the image is 


$$
P\left(k_{x}, k_{y}, z\right)= \begin{cases}P_{\mathrm{obj}}\left(k_{x}, k_{y}, z\right), & \text { if } \frac{\left(\left(k_{x}-r_{x}\right)^{2}+\left(k_{y}-r_{y}\right)^{2}\right)^{\frac{1}{2}}}{k_{i}}<\frac{\mathrm{NA}_{z}}{n_{i}} \\ 0, & \text { otherwise. }\end{cases}
$$

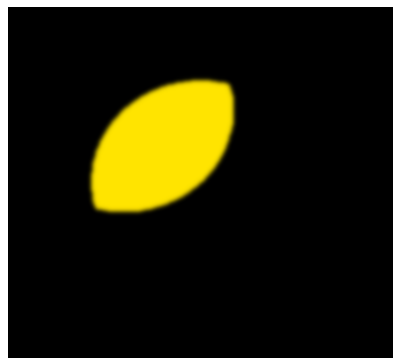

Fig. 8. Chopped pupil amplitude as a result of two aperture with $25 \%$ overlap. The overlap region shows the permitted light.
Slice XY

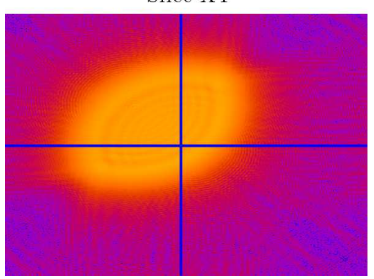

Slice XZ

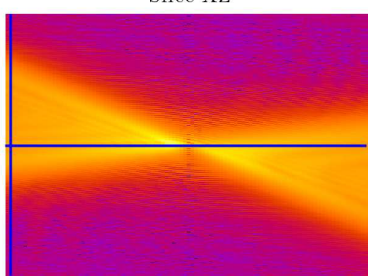

Fig. 9. Evidence of the resemblance between the OOFHs and the aperture shape for a MACROscope. Left: The far side out-of-focus lateral slice of the theoretically modeled PSF on a log scale, obtained from a 75\% chopped pupil of Fig. 8. Right: Position of the out-offocus slice along the optical axis.

cropped from the periphery of the field. Our observation is that the model is able to mimic very well the condition of lateral variance of the PSF within the field. However, the lateral full width at half maximum (FWHM) of the model is smaller than the experimentally obtained image's lateral FWHM. This difference can be explained by the fact that the size of the microsphere used is significantly large to be considered as a point source.

Our future work is aimed at enhancing this initial PSF model further with newer acquisitions on different systems. Following in this direction, the eventual goal is to correct this field aberration in the observed specimen images. Given the fact that the MACROscope works under a variable zoom, in the absence of microsphere images, the effective NA of the optical mount is unknown, and this probably has to be estimated during restoration.

\section{REFERENCES}

[1] P. Sendrowski and C. Kress, "Arrangement for analyzing microscopic and macroscopic preparations," WO 2009/04711, Apr. 2009, PCT/EP2008/062749. 1

[2] W. Yu, "Practical anti-vignetting methods for digital cameras," IEEE Trans. on Consumer Electronics, vol. 50, no. 4, pp. 975983, Nov. 2004. 1
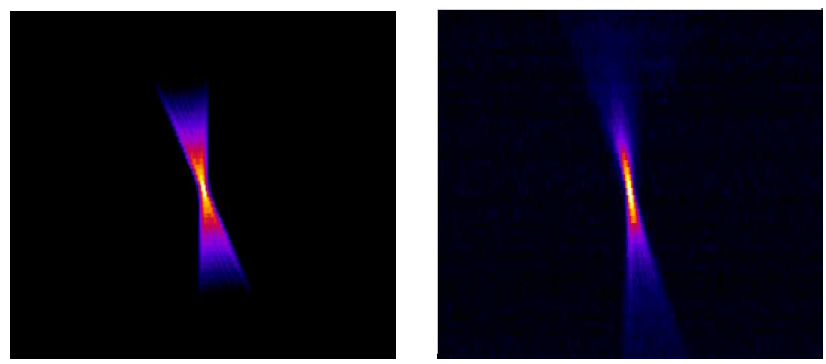

Fig. 10. Left: Axial intensity projection of a theoretically modeled PSF with a $25 \%$ overlap. Right: Projection of a $4 \mu \mathrm{m}$ fluorescent bead on a log scale (C)Imaging Center, IGBMC, France.). The radial sampling is $998.3 \mathrm{~nm}$ and the axial sampling is $1000 \mathrm{~nm}$. The beads were imaged using a Leica ${ }^{\mathrm{TM}}$ MacroFluo ${ }^{\mathrm{TM}}$ at a zoom of $1.6 \times$, along the periphery of the field.

[3] Y. Zheng, S. Lin, C. Kambhamettu, J. Yu, and S. B. Kang, "Single-Image Vignetting Correction," IEEE Trans. on Pattern Analysis and Machine Intelligence, vol. 31, no. 12, pp. 22432256, Dec. 2009. 1

[4] S. Lyu, "Single image vignetting correction with natural image statistics in derivative domains," in Proc. IEEE International Conference on Image Processing, Sept. 2010, pp. 3529-3532. 1

[5] P. Pankajakshan, Blind Deconvolution for Confocal Laser Scanning Microscopy, Ph.D. thesis, Université de Nice SophiaAntipolis, Dec. 2009. 2

[6] R. Kingslake, Optics in Photography, SPIE Publications, June 1992. 2

[7] Sidney F. Ray, Applied Photographic Optics: Lens and Optical Systems for Photography, Focal Press, third edition, Apr. 2002. 2

[8] M. Born and E. Wolf, Principles of Optics, Cambridge U. Press, 1999. 3

[9] S. F. Gibson and F. Lanni, "Diffraction by a circular aperture as a model for three-dimensional optical microscopy," J. Opt. Soc. Am. A, vol. A6, pp. 1357-1367, 1989. 3

[10] Y. Hiraoka, J. W. Sedat, and D. A. Agard, "Determination of three-dimensional imaging properties of a light microscope system," Biophys. J., vol. 57, pp. 325-333, Feb. 1990. 3

[11] P. A. Stokseth, "Properties of a defocused optical system," J. Opt. Soc. Am. A, vol. 59, pp. 1314-1321, Oct. 1969. 4 